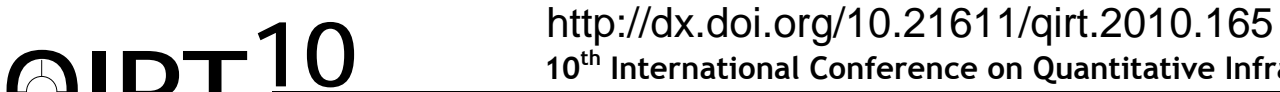 \\ $10^{\text {th }}$ International Conference on Quantitative InfraRed Thermography \\ July 27-30, 2010, Québec (Canada)
}

\section{Time-Frequency Analysis of Skin Temperature in a Patient with a Surface Tumor Monitored with Infrared Imaging}

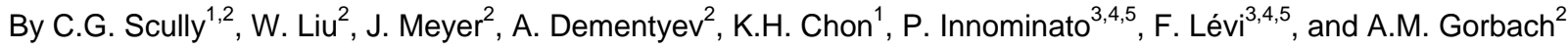 \\ ${ }^{1}$ Worcester Polytechnic Institute, Worcester, MA, USA \\ ${ }^{2}$ National Institutes of Health, Bethesda, MD, USA, gorbacha@mail.nih.gov \\ 3 INSERM, U776 «Biological rhythms and cancers», Paul Brousse Hospital, Villejuif, France, francis.levi@inserm.fr \\ ${ }^{4}$ Univ Paris Sud 11, SO776, Orsay, France \\ ${ }^{5}$ AP-HP, Department of Medical Oncology, Chronotherapy Unit, Paul Brousse Hospital, Villejuif, France
}

\begin{abstract}
Infrared thermography was used to assess skin temperature in a patient with a surface tumor across a range of frequencies relating to blood flow fluctuations. Using a high acquisition rate of image capture and wavelet analysis we were able to study temperature dynamics from low frequencies related to vasomotion $(\sim 0.01 \mathrm{~Hz})$ up to the cardiac rate $(\sim 1 \mathrm{~Hz})$. We show that IR imaging can be used to monitor skin temperature in spatial, time, and time-frequency domains allowing the study of coupling between different microvascular territories.
\end{abstract}

\section{Introduction}

Blood flow in the microcirculation can often be assessed by use of optical imaging techniques such as Laser Doppler and Laser Speckle. These techniques allow for frequency analysis of blood flow fluctuations across a spectrum of frequencies that are related to NO-independent endothelial activity (Range V: $0.005-0.0095 \mathrm{~Hz}$ ), NO-dependent endothelial activity (Range VI: $0.0095-0.021 \mathrm{~Hz}$ ), neurogenic (sympathetic) activity (Range IV: $0.021-0.052 \mathrm{~Hz}$ ), myogenic activity (Range III: $0.052-0.145 \mathrm{~Hz}$ ), respiration (Range II: $0.145-0.6 \mathrm{~Hz}$ ), and heart rate (Range 1: $0.6-2 \mathrm{~Hz}$ ) [1]. Use of frequency analysis techniques allows the study of the contribution of each physiological component to the overall local blood flow regulation. Previously, skin temperature has also been used to study these components, but the low-pass filter characteristics of the skin attenuate higher frequency temperature changes. Bandrivskyy et al. measured skin temperature with thermistors along with simultaneous laser Doppler measurements and used Wavelet Phase Coherence (WPC) to show the effects of blood flow on skin temperature up to the $0.1 \mathrm{~Hz}$ range, at which point skin temperature oscillations contributed to blood flow are masked by noise [2]. Infrared (IR) imaging reflects skin temperature through radiation following conduction and convection of heat to the surface of the skin, but does not rely on inertia such as a thermistor. Therefore, with IR measurement it is possible to assess the temperature of living tissue at more depth than the surface. Because of this there is an expectation that IR imaging would allow the analysis of lower and higher frequencies through the skin. Gorbach et al. used IR imaging to study blood flow oscillations in the kidney using wavelet analysis [4], and during intraoperative monitoring of human brain with an IR camera heart rate in individual vessel segments on the cortex was monitored [5]. Garbey et al. measured the cardiac pulse using IR through the skin, with the consequence of filtering out low frequency oscillations relating to blood flow [3].

Tumor microvasculature is known to be atypical in structure as well as function. The exact nature of vascular abnormalities that may be present in a specific tumor at a specific stage is not well known, but may include increased angiogenesis, non-specific flow directions, and stagnant flow [6]. These differences in structure and function can diminish blood flow regulation in tumors and lead to decreased effectiveness of medication [6]. The oscillatory nature of the microvasculature in tumors compared to normal tissue has not been well characterized. The objective of this work is to assess the ability of an IR camera to monitor skin temperature fluctuations as related to blood flow and compare differences in temperature patterns between skin regions in a patient presenting with a surface tumor. Time-frequency analysis between regions of interest within and outside of the tumor boundaries was performed.

A thermally conductive region, described in [7], around the bridge of the nose has previously been associated with brain temperature stemming from the hypothalamic thermoregulatory center. A secondary objective of this work is to assess stability and differences in brain tunneling temperature (BTT) between the right and left side of the nose monitored with IR imaging.

\section{Methods}

\subsection{Image Acquisition}

IR images were acquired with an FLIR SC7700 camera (3-5 micron wavelength, 640x512 pixels/image, 14 bits) with a temperature resolution of $0.017^{\circ} \mathrm{C}$ at an acquisition rate of $10 \mathrm{~Hz}$. CNUC ${ }^{\mathrm{TM}}$ is an FLIR calibration process that adjusts camera properties during imaging to correct for room temperature. This function was enabled during the patient imaging procedure. The patient gave written informed consent for this non-invasive procedure. The patient was seated in a chair and asked to look on a color target above the camera lenses and stay stationary for 25 minutes during imaging. A total of 15,000 images were collected. Pixel size was determined to be $\sim 0.62 \mathrm{~mm} /$ pixel by measuring known distances in the image. 
The IR camera was placed $\sim 1.5 \mathrm{~m}$ from the patient and was focused on the face of the patient, where a surface tumor was present on the left side of the nose. The patient had widespread metastatic clear cell cancer of the kidney. A $3 \times 3 \mathrm{~cm}^{2} \mathrm{skin}$ metastasis was located in the left naso-genian area of the upper face. As a result of the well known high vascularization of such tumor type, which releases a high amount of Vascular Endothelial Growth Factor, there were numerous visible surface vessels at the tumor surface, in the periphery. The occurrence of necrosis was responsible for an open wound in the central part of tumor.

Post-experiment, two black-body (BB) calibration sessions were performed to assess the noise characteristics of the camera. In the first session, the camera was set at an acquisition rate of $10 \mathrm{~Hz}$ and the $\mathrm{BB}$ source was imaged at $30{ }^{\circ} \mathrm{C}$ for 30 minutes with CNUC enabled. A 200x200 pixel region within the center of the black body was selected for further temporal noise analysis of the IR camera. The selected 200x200 pixel region was divided into $1,600-5 \times 5$ pixel regions of interest (ROIs). The mean power spectral density (PSD) of each ROI was calculated using a Welch periodogram with a window length of 4,096, 50\% overlap, and Fast Fourier Transform (FFT) length of 4,096. The second session was performed with the camera at an acquisition rate of $2 \mathrm{~Hz}$, BB source at $30{ }^{\circ} \mathrm{C}$ for 25 minutes, and CNUC disabled. A 200x200 pixel region was subdivided into 1,600-5x5 pixel ROIs, and the PSD was calculated for each ROI with window length of $1,024,50 \%$ overlap, and FFT length of 1,024 .

\subsection{Image Alignment and ROI Selection}

The first 4 minutes and 30 seconds were removed because of significant motion artifacts related to the patient breathing. After this point the patient breathed primarily through the right (non-tumor side) nostril, and motion was reduced. The remaining images were aligned using a least-squares alignment method from the Statistical Parametric Mapping toolbox [8] using the first image, after removal of the initial 4 minutes and 30 second period, as the reference frame.

One 40x45 pixel region was selected on the non-tumor side of the face (control area) and another on the tumor side, figure 1. Each of these was further broken up into 72-5x5 pixel ROIs. Futher, additional $5 \times 5$ ROIs were selected corresponding to various facial features including a region of the tumor that was partially wet (hereafter referred to as Tumor Wet), inner corner on the left and right bridge of the nose (potentially reflecting brain tunneling temperature or BTT), and background room temperature. We selected the hottest pixel on the left and right side of the nose in the described region in [7] as the center of an ROI to monitor BTT. A map of the selected ROIs on the patient is shown in figure 1 . The background room temperature location is not shown in figure 1 . This was selected from the upper corner above the tumor side of the face, where the impact of breathing and body heat should be at a minimum.

(a)

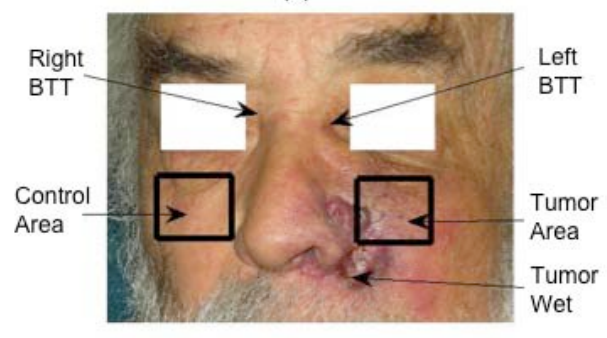

(b)

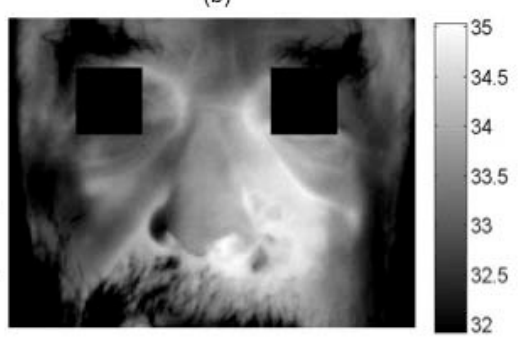

Fig. 1. Visible light (a) and IR image (b) of the patient with marked ROIs on the visible image. The two boxes denote the regions selected to represent the tumor and control sides of the face, which were each further broken up into 72 equally sized ROIs. Scale bar is temperature in ${ }^{\circ} \mathrm{C}$.

\subsection{Time-Frequency Analysis}

For each ROI, the $5 \times 5$ pixel time-series were averaged together and then detrended with a second order polynomial followed by subtraction of the mean. Wavelet analysis was performed using an Adaptive Wavelet Transform (AWT) proposed to be used for blood flow oscillation analysis by Tankanag et al. to minimize the edge effect inherent to the continuous wavelet transform [9]. The continuous wavelet transform is shown in Eq. (1), where $x(u)$ is the signal and $Y_{s, t}$ is the analyzing wavelet that is shifted in location and scale to generate the time-frequency representation.

$$
W_{n}(s, t)=\int Y_{s, t}(u) x(u) d u
$$

The integral of the analyzing wavelet at each time point must approach 0 under the admissibility condition. This does not hold true when the wavelet transform is at the edges of the signal, and wavelet results at these locations can be erroneous. The AWT adjusts the analyzing wavelet at the edges by adding a parameter $R$ to satisfy the admissibility condition, Eq. (2) and (3).

$$
\bar{Y}_{s, t}\left(t^{\prime}, t, s\right)=\left[h\left(\frac{t^{\prime}-t}{s}\right)-R(s, t)\right] \Phi\left(\frac{t^{\prime}-t}{s}\right) G\left(t^{\prime}\right)
$$




$$
R(s, t)=\frac{\int_{-\infty}^{\infty} h\left(\frac{t^{\prime}-t}{s}\right) \Phi\left(\frac{t^{\prime}-t}{s}\right) G\left(t^{\prime}\right) d t^{\prime}}{\int_{-\infty}^{\infty} \Phi\left(\frac{t^{\prime}-t}{s}\right) G\left(t^{\prime}\right) d t^{\prime}}
$$

$G$ is a vector representing the locations near the edges where the analyzing wavelet is adjusted and $h$ and $\Phi$ are two components of the analyzing wavelet. In this study, the Morlet wavelet was used as the analyzing wavelet with a Mother wavelet parameter of 6 . Further details on the Morlet wavelet can be found in [10]. For a discrete signal the AWT generates a value for each time and input scale. Scales, $s$, for wavelet analysis were chosen to correspond to frequencies from 0.005 to $2 \mathrm{~Hz}$. To generate a representation of the overall power for each frequency range of interest the Scale-Averaged Power (SAP) was computed as Eq. (4).

$$
\bar{W}_{n}^{2}=\frac{d j d t}{C} \sum_{j=j_{1}}^{j_{2}} \frac{\left|W_{n}\left(s_{j}\right)\right|^{2}}{s_{j}}
$$

$C$ is a predetermined reconstruction factor $\left(0.776\right.$ for the Morlet wavelet with $\left.\omega_{0}=6\right), d j$ is the scale resolution, $d t$ is the sampling times, $s_{j}$ is the current scale, and $j_{1}$ and $j_{2}$ are the border scales for each frequency region. The scale resolution was set at 0.0625 . Details of the calculation to SAP can be found in [10]. SAP values quantify the magnitude of frequency content across each frequency band of interest, where higher values represent an increased contribution of that particular frequency range to the overall dynamics of the signal.

For the tumor and control region, WPC, as outlined by Bandrivskyy et al., was applied to the wavelet spectrums between a center ROI and all other ROI's in that region [2]. WPC values range between 0 and 1, where 0 represents no phase coherence between the signals and 1 represents the highest amount of possible phase coherence. This produced a total of 71 WPC values at each frequency output from the wavelet transform for the tumor and control side. WPC values were averaged across each frequency range. WPC was also calculated between 30 pairs of random Gaussian noise at the same signal length as the imaging period analyzed and sampling frequency of $10 \mathrm{~Hz}$. For each ROI and each frequency range, WPC was compared to the mean + SD of the averaged Gaussian WPC value across the corresponding frequency range to determine significance.

The respiration rate $(R R)$ was determined by finding the peak wavelet power within the range of 0.145 and $0.4 \mathrm{~Hz}$. A location under the non-tumor nostril, where the effects of breathing were the most obvious, was taken to be the true RR. Percent errors between the respiration rate measured from other locations and the true RR were calculated as given in Eq. (5), where MR is the measured RR and TR is the true RR.

$$
\frac{|M R-T R|}{T R} \times 100=\% \text { Error }
$$

A time-averaged value for the maximum peak in the HR range was found by selecting the time-averaged wavelet power between 0.6 and $2 \mathrm{~Hz}$, making the region linear by taking the $\log _{10}$ of the wavelet powers, linearly detrending the array, and selecting the frequency corresponding to the maximum detrended power. A location to validate HR could not be obtained from the experimental conditions.

\subsection{Statistical Analysis}

Differences in mean temperature, SAP, and WPC values between the control and tumor side were determined with the Mann-Whitney Rank Sum Test for non-parametric data, $p<0.05$ considered significant, using each $5 \times 5 \mathrm{ROI}$ in the control and tumor region as an event. Statistical differences in the number of significant WPC values above the white-noise threshold in the control and tumor region were determined using the Pearson Chi-Square test.

\section{Results}

\subsection{Noise Characteristics of IR Camera}

The averaged log-log power spectrum over all ROls from both camera BB sessions is shown in figure 2 for the frequency range of interest from 0.005 to $1 \mathrm{~Hz}$. Two distinct regions of the spectrum were found for the first calibration session imaged at $10 \mathrm{~Hz}$ with $\mathrm{CNUC}^{\mathrm{TM}}$ enabled (dashed line in figure 2), and the slope for each of these regions was determined by finding the best-fit linear line using a least-squares approach. Between 0.005 and $0.2 \mathrm{~Hz}$ the slope was calculated to be -1.56 , in the range of brown noise, and between 0.2 and $2 \mathrm{~Hz}$ the slope was found to be -0.18 , close to white noise. A Brown noise spectrum in our range of interest indicates that the temporal images may have an underlying nonstationary component from the imaging system itself. When CNUC ${ }^{\mathrm{TM}}$ was disabled and imaging of the BB was performed at $2 \mathrm{~Hz}$ (thin line) the power in the low frequency range is less than that of the first BB session by almost a full order of magnitude at times. The power for the second BB session stays relatively flat across all frequencies. 


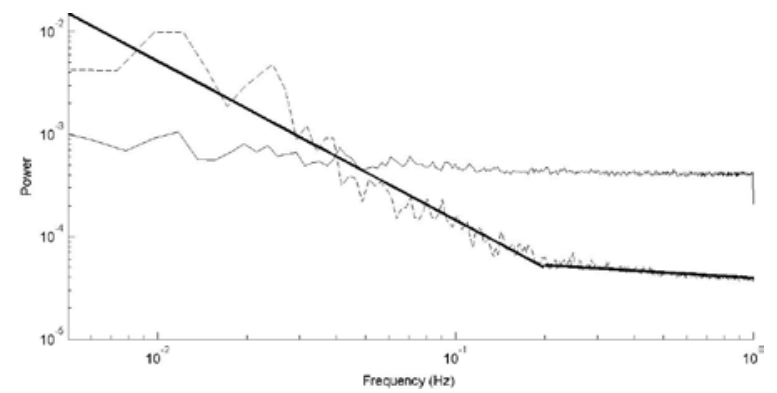

Fig. 2. Log-log PSD's of BB imaging from 0.005 to $1 \mathrm{~Hz}$. The first session sampled (dashed line) at $10 \mathrm{~Hz}$ has $2 \mathrm{best}$-fit linear lines (thick lines) representing brown noise $(0.005$ to $0.2 \mathrm{~Hz})$ and noise close to white $(0.2$ to $1 \mathrm{~Hz})$. The second session sampled at $2 \mathrm{~Hz}$ (thin line) with CNUC ${ }^{T M}$ function disabled has noise diminished by almost a full order of magnitude in the frequency regions related to endothelial activity $(0.005$ to $0.2 \mathrm{~Hz})$.

\subsection{Time-frequency Analysis of Skin Temperature}

\subsubsection{Skin Temperature Fluctuations in Control and Tumor Regions}

After alignment, the skin temperature was determined from the FLIR camera absolute units using a fourth-order polynomial determined with the FLIR calibration system. Figure 3 shows an example 3-minute segment of the time-series from a control ROI before and after alignment, as well as an example background room temperature.

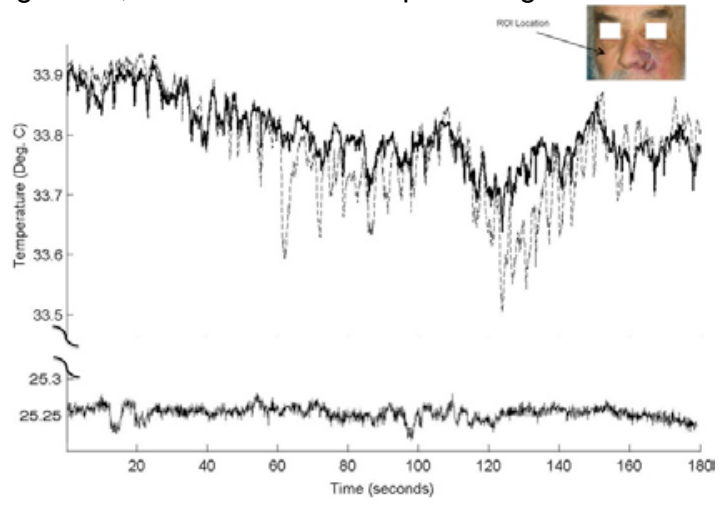

Fig. 3. Example $5 \times 5$ pixel averaged 3-minute portion of the time-series with overlapped before alignment (dashed-line) and after alignment (thick-line). Sudden dramatic changes in temperature are diminished after alignment. The thin black line at the bottom represents background room temperature as measured from the IR camera in a location away from the body.

The ROI averaged temperatures for the control and tumor regions, BTT for the right and left sides, and background room temperature are shown in Table 1. Mean temperature between the control and tumor region was significantly different.

Table 1. Mean $\pm S D$ temperature $\left({ }^{\circ} \mathrm{C}\right)$ for each $\mathrm{ROI}$ shown in figure 1 plus background room temperature. $\alpha$ denotes significance between the tumor and control regional temperature means, $p<0.05$.

\begin{tabular}{|c|c|c|c|c|c|c|}
\hline & Control & \multicolumn{2}{|c|}{ Tumor } & \multicolumn{2}{|c|}{ BTT } & Left \\
\cline { 2 - 7 } & ROI Average & ROI Average & Wet & Right & Background \\
\hline Temp. $\left({ }^{\circ} \mathrm{C}\right)$ & $33.29 \pm 0.32^{\alpha}$ & $34.33 \pm 0.31^{\alpha}$ & $33.77 \pm 0.12$ & $34.03 \pm 0.08$ & $34.46 \pm 0.05$ & $25.26 \pm 0.26$ \\
\hline
\end{tabular}

The AWT was computed for each $5 \times 5$ pixel averaged ROI time series. A representative time-frequency spectrum generated from the AWT is shown in figure $4 \mathrm{a}$. 


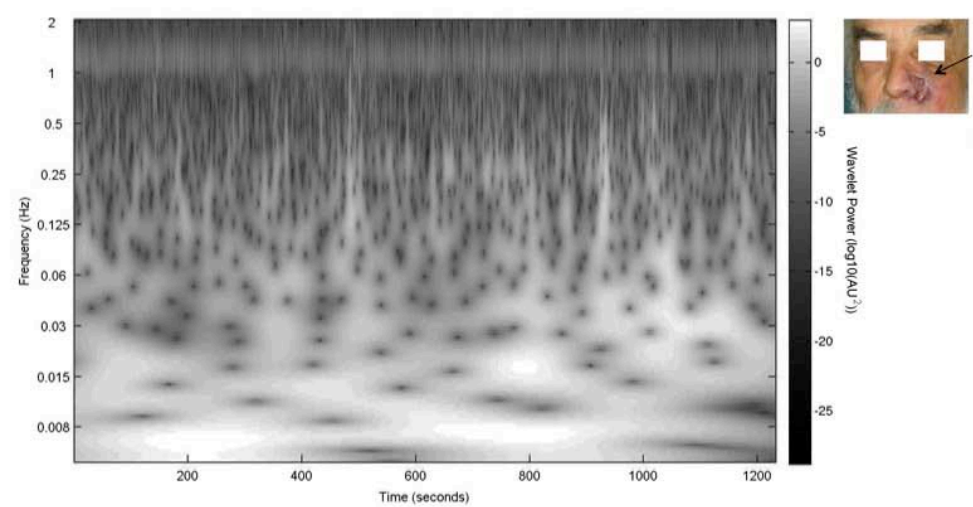

(b)

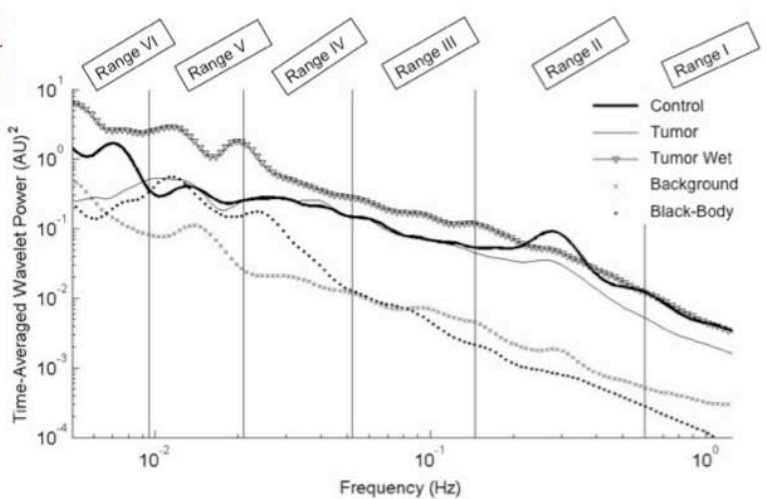

Fig. 4. (a)Sample time-frequency representation of skin temperature generated by the adaptive wavelet transform from a

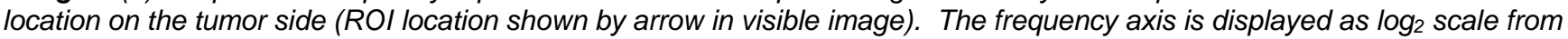
0.005 to $2 \mathrm{~Hz}$, and wavelet power is displayed as $\log _{10}$ scale. Time-frequency information is obtained from very low frequency blood flow oscillations up through the cardiac rate, which can be seen as a light band across time just above $1 \mathrm{~Hz}$. (b) Log-log scale plot of mean wavelet power for a control ROI, tumor ROI, tumor wet ROI, background room temperature, and BB calibration file from 0.005 to $1.25 \mathrm{~Hz}$. Six frequency ranges make up the known components of local blood flow regulation.

Figure 4b shows the log-log plot of the time-averaged wavelet power calculated for a control ROI, tumor ROI, tumor wet, background room temperature, and BB calibration. Each ROI has power above that of the background room temperature except in the very low frequency region between 0.005 and $0.007 \mathrm{~Hz}$. Distinct peaks in the background noise can be seen in some of the frequency regions, especially respiration. These may be caused by periodic heating of the air coming from the body instead of a true representation of room temperature. The high power in the BB calibration profile between 0.005 and $0.2 \mathrm{~Hz}$ can be seen on the same order of magnitude as the control and tumor ROIs.

SAP was calculated from the wavelet time-frequency representation using Eq. (4) for each frequency range, and the mean SAP was taken to represent the power in each range across all time. Table 2 shows the mean SAP in arbitrary units squared $\left(A b . U^{2}\right)$ for each frequency range plus the full range from 0.005 to $2 \mathrm{~Hz}$ at each location calculated from absolute camera units. For all frequency bands expect range IV the control region had significantly higher SAP than the tumor region. In higher frequencies this may be an artifact of the effect of breathing, but in low frequencies relating to blood flow this may be a sign of a more active microcirculatory system in the control region.

Table 2. SAP $\left(A b . U^{2}\right)$, mean $\pm S D$, calculated from the AWT across each frequency range related to blood flow for the control region, tumor region and Tumor Wet ROI. There is no SD for the Tumor Wet ROI because it is a single location, but the wet ROI has high SAP values compared to the rest of the tumor region. $\alpha$ denotes significant differences between the control and tumor regions, $p<0.05$.

\begin{tabular}{|l|c|c|c|}
\hline \multirow{2}{*}{$\begin{array}{l}\text { Frequency Ranges } \\
\left(\mathrm{Ab} . \mathrm{U}^{2} \star 10^{-3}\right)\end{array}$} & Control & \multicolumn{2}{|c|}{ Tumor } \\
\cline { 2 - 4 } & ROI Average & ROI Average & Wet \\
\hline Range I & $1.04 \pm 0.3^{\alpha}$ & $0.63 \pm 0.3$ & 1.06 \\
\hline Range II & $3.81 \pm 1.2^{\alpha}$ & $1.95 \pm 0.7$ & 3.28 \\
\hline Range III & $2.23 \pm 1.0^{\alpha}$ & $1.80 \pm 0.9$ & 2.88 \\
\hline Range IV & $2.12 \pm 1.2$ & $2.07 \pm 1.6$ & 3.09 \\
\hline Range V & $1.36 \pm 0.7^{\alpha}$ & $1.14 \pm 1.0$ & 4.35 \\
\hline Range VI & $1.13 \pm 0.6^{\alpha}$ & $0.94 \pm 1.1$ & 2.93 \\
\hline Range I-VI & $11.7 \pm 4.6^{\alpha}$ & $8.52 \pm 5.5$ & 17.6 \\
\hline
\end{tabular}

\subsubsection{Non-Contact Monitoring of Vital Signs with IR Camera}

\subsubsection{Respiration Rate}

Respiration was most visible on the control side of the face where the physical impact of breathing is most significant. RR was measured to vary between 0.2 and $0.35 \mathrm{~Hz}$. Underneath the right nostril of the patient the physical effects of breathing were most obvious, and from this location it was possible to determine the mean respiratory rate to be $16.9 \pm 1.5 \mathrm{BPM}$ by first finding the peak wavelet power in the linearly detrended frequency range between $0.145 \mathrm{and} 0.4 \mathrm{~Hz}$, removing any peaks that were at the minimum or maximum, and then averaging the derived respiratory time-series. Peaks at the maximum or minimum frequencies are caused by diminished wavelet power from respiration at those time instances, and therefore it is acceptable to remove their contribution to the overall RR. The derived RR time-series is shown in figure 
$5 a$, and figure $5 b$ shows the Fast Fourier Transform (FFT) spectrum across the respiratory range. The RR from the location shown in figure 5 was taken to be the "true" RR to compare RRs obtained from other locations with. The variance in RR can be attributed to the patient undergoing spontaneous breathing during imaging.

(a)

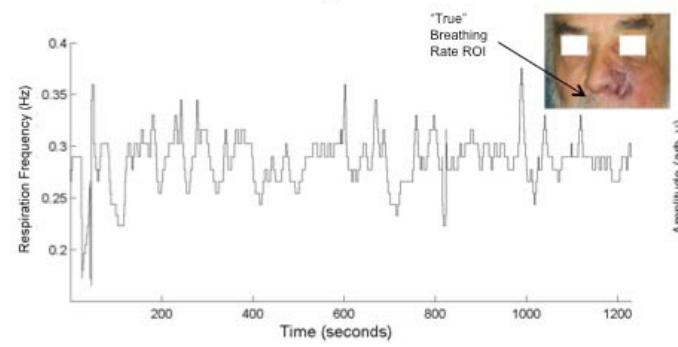

(b)

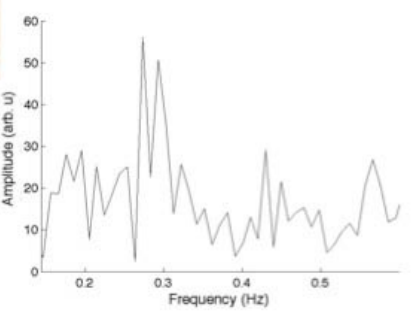

Fig. 5. (a) Time-series of "true" respiration rate extracted from the wavelet time-frequency spectrum. The arrow on the visible image in the upper right corner indicates the location for the "true" breathing rate. (b) 1024-point FFT of the "true" breathing rate temperature signal between 0.145 to $0.6 \mathrm{~Hz}$. Two peaks relating to respiration can be seen on the FFT at $0.273 \mathrm{~Hz}$ and

$0.293 \mathrm{~Hz}$. Use of only the FFT does not tell us about changes in respiration over time, while the AWT allows us to find a respiration peak at each time point.

Table 3 shows the mean RR found using the same method described above for the tumor and control regions and percent error with the "true" RR. Differences between the "true" RR, control and tumor values can be attributed to inconsistent power across the respiratory range. In figure 4 it can be seen that the magnitude of power in the respiration range from 0.25 to 0.3 $\mathrm{Hz}$ varies across time, producing inconsistent RR compared with directly monitoring breathing with the "true" RR location.

Table 3. Measured $R R$ in BPM, mean $\pm S D$, and percent error from "true" $R R$. The control side has a mean $R R$ closer to the "true" RR compared with the tumor side of the face.

\begin{tabular}{|c|c|c|}
\hline & Mean RR \pm SD & $\%$ Error \\
\hline "True" & $16.9 \pm 1.5$ & - \\
\hline Control & $15.0 \pm 0.44$ & 11.2 \\
\hline Tumor & $13.3 \pm 0.19$ & 21.3 \\
\hline
\end{tabular}

\subsubsection{Heart Rate}

Measurement of HR could be performed on specific locations on the face. The HR range contributed to blood flow oscillations is large, from 36 to 120 BPM. From wavelet analysis of locations on the tumor side of the face, it was determined that HR was approximately $1.06 \mathrm{~Hz}$, or $63.6 \mathrm{BPM}$. Figure 4 shows a light band just above $1 \mathrm{~Hz}$ representing the HR. Locations that had HR within 55 and 70 BPM determined using the method described in section 2.3. were considered to be candidates for representing HR. Six such locations were found on the tumor side of the face, and one location was found on the control side of the face.

Many small vessels existed in the area on the tumor where HR was found, as examined from a visible light image. Vessel size where HR was found on the tumor region was determined to be $\sim 0.8 \mathrm{~mm}$ in diameter. Attenuation of higher frequencies through the skin is diminished from these surface vessels, possibly allowing HR to be measured here.

\subsubsection{Brain Tunneling Temperature}

The AWT was calculated for BTT, and SAP values for the right and left side are shown in Table 4. In comparison with Table 2, mean SAP values for BTT are less than those for other ROIs on the face for both sides, representing relatively stable BTT. Substantial differences are not seen between the two sides.

Table 4. Scale-averaged powers $\left(A b . U^{2}\right)$ for BTT computed from the adaptive wavelet transform.

\begin{tabular}{|l|c|c|}
\hline \multirow{2}{*}{$\begin{array}{l}\text { Frequency Ranges } \\
\left(\text { Ab. } U^{2} * 10^{-3}\right)\end{array}$} & Right & Left \\
\cline { 2 - 3 } Range III & 1.11 & 1.15 \\
\hline Range IV & 0.98 & 1.24 \\
\hline Range V & 0.36 & 0.48 \\
\hline Range VI & 0.27 & 0.30 \\
\hline
\end{tabular}

\subsection{Wavelet Phase Coherence}

WPC was calculated between the center ROI in the control and tumor side and all other ROIs on their respective side for a total of 71 locations. In [2] it was shown that WPC increases for uncorrelated white noise as the frequency tends towards $0 \mathrm{~Hz}$. The coherence across each frequency range was averaged for each ROI, and compared to the mean $+\mathrm{SD}$ of the WPC for the white noise significance test in that range, figure $6 \mathrm{a}$. A map of the WPC averaged across range $\mathrm{V}$ is shown 
in figure $6 \mathrm{~b}$ for both sides of the face, and significant values above the white-noise threshold for range $V$ are marked with an $X$ in figure $6 b$.

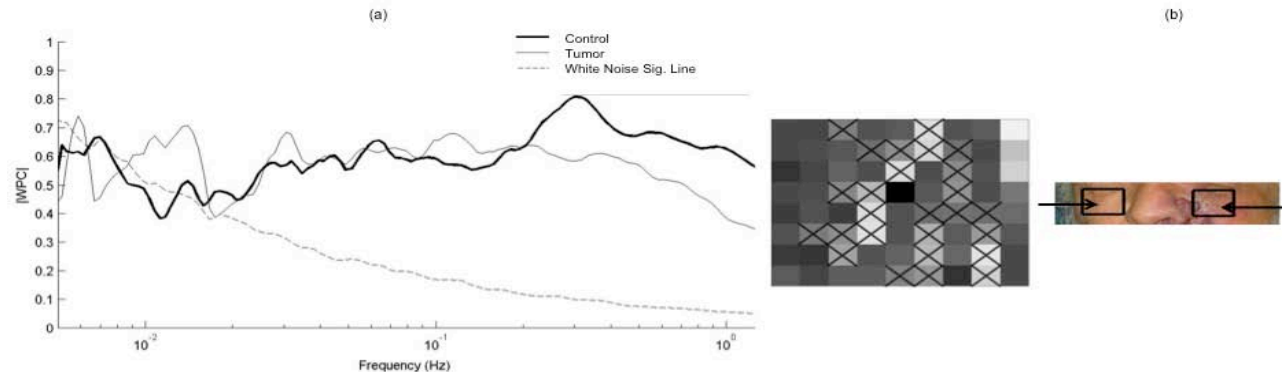

Fig. 6. (a) Time-averaged WPC magnitude averaged across all ROIs for the tumor (thin line) and control (thick line) regions. A white noise-significance line (dashed line) is shown to increase at low frequencies. In the higher frequency ranges both regions have average WPC above that of the threshold, but this difference diminishes at low frequencies. (b) Map of the averaged WPC across frequency Range $V$ for each ROI with the center ROI for it's respective side (dark black square in the center of each map is the central ROI) on the tumor and control side of the face. An ' $X$ ' represents the WPC for that ROI with the center $\mathrm{ROI}$ is greater than the white noise significance line in frequency range $\mathrm{V}$.

Table 5.a. shows the total number of ROIs that had WPC higher than that of the white noise threshold line at four frequency ranges. Mean WPC values for four frequency ranges are shown in Table 5.b. for the control and tumor side.

Table 5. (a) Total number of WPC significant ROIs out of 71 possible as judged by the white noise significance test for the control and tumor side for frequency ranges III-VI. (b) Average WPC for frequency ranges III-VI between the center ROI and all other ROIs in the control and tumor regions. $\beta$ denotes significance for the Pearson Chi-Square test, and $\alpha$ denotes significance determined by the Mann-Whitney Rank Sum Test between the control and tumor side, $p<0.05$. For both methods, the control side of the face has decreased phase coherence compared to the tumor side in the frequency range related to NO-dependent endothelial activity, Range $\mathrm{V}$.

(a)

\begin{tabular}{|c|c|c|}
\hline & Control & Tumor \\
\hline Range III & 68 & 69 \\
\hline Range IV & 65 & 61 \\
\hline Range V & $30 \boldsymbol{\beta}$ & 47 \\
\hline Range VI & 24 & 19 \\
\hline
\end{tabular}

(b)

\begin{tabular}{|c|c|c|}
\hline & Control & Tumor \\
\hline Range III & $0.59 \pm 0.23$ & $0.63 \pm 0.24$ \\
\hline Range IV & $0.56 \pm 0.22$ & $0.58 \pm 0.22$ \\
\hline Range V & $0.46 \pm 0.21 \boldsymbol{\alpha}$ & $0.57 \pm 0.21$ \\
\hline Range VI & $0.60 \pm 0.16$ & $0.56 \pm 0.16$ \\
\hline
\end{tabular}

There is increased WPC between tumor locations in the low frequency blood flow range $\mathrm{V}$ as shown by an increased number of ROls that have coherence greater than the white noise threshold, as well as an increase in the overall mean value.

\section{Discussion}

Spectral analysis for the $10 \mathrm{~Hz}$ sampled BB with CNUC ${ }^{\mathrm{TM}}$ function enabled showed Brownian motion with high power between 0.005 and $0.2 \mathrm{~Hz}$, but diminished power after $0.2 \mathrm{~Hz}$ in a pattern closer to white noise. Noise levels, as determined by the BB source, were on the same order of magnitude as the physiological signal in the area of $0.01 \mathrm{~Hz}$ (figure 5) with these settings. With $\mathrm{CNUC}^{\mathrm{TM}}$ disabled, low frequency noise was decreased by almost a full order of magnitude (figure 2). It appears that the $\mathrm{CNUC}^{\mathrm{TM}}$ procedure may be the cause of high power in low frequencies by making room temperature compensations during the imaging session.

Using wavelet analysis we are able to take a single $5 \times 5$ pixel ROI time-series of temperature and extract it into a time-frequency representation giving us information about the current frequency content of temperature changes at any sampled time point. Figure 4a shows an example of the vast amount of information present in one ROI from the tumor side of the face. Here we can see content from very low frequencies related to non-NO dependent endothelial activity up to the heart rate. We can then separate each frequency band using SAP, and compare the content within each band in the context of the whole time-frequency plot or against the same band from other signals.

Differences in SAP as well as WPC between locations inside and outside of the tumor boundaries suggest differences in microcirculation between the tumor and control sides. The control region had significantly higher SAP in frequency ranges related to both NO-dependent and NO-independent endothelial activity, $\mathrm{V}$ and $\mathrm{VI}$. These regions appear to be more active in the control region than then tumor region. Increased SAP in the high frequency ranges, IV, V, and VI, also existed. The impact of breathing on the control side is most likely the source of increased power in high frequency ranges, and reasonable values of RR could be obtained from the control locations, Table 3. Differences in RR values between tumor and control locations may be attributed to changes in wavelet power over time. Only time points that had a peak in a 
reasonable respiratory range were included in the average of RR over the whole time period. This led to differences in time points averaged together to generate the mean RR's shown in Table 3. The wet tumor ROI had high SAP, and the increase at this location may be attributed to effects of evaporative cooling [4]. BTT sites had relatively low SAP on the control and tumor side, showing more stability in temperature at these locations. This was expected since BTT is thought to be an indirect measure of brain temperature, which should stay relatively stable.

Increased WPC was present in the control location for frequency range $\mathrm{V}$, related to NO-dependent endothelial activity [1]. This was measured and found to be significant two ways: by finding the total number of ROIs with WPC above the threshold line and by calculating the mean WPC across all ROIs. For both sides, the ROIs close to the center ROI had a higher percentage of significant WPC at range $\mathrm{V}$. On the control side 14 out of 26 ROls within a 2 ROI boundary around the center had significant WPC, while 16 out of 45 ROIs were significant outside of the 2 ROI boundary. For the tumor side, 21 out of 26 ROls were significant inside of the same boundary, and 21 out of 45 were significant outside. Increased WPC close to the center ROI is expected because heat from a source will dissipate and spread across the skin. Therefore, local regions should be well correlated at frequencies relating to blood flow with some phase-lag depending on their distance from a specific heat source. The skin surface may have many heat sources stemming from various underlying microvascular territories. An increased number of sources would increase the complexity of the temperature signal at each skin position and correlations between regions would most likely decrease. This may be the reason why phase coherence in range $\mathrm{V}$ is diminished on the control skin region. Where the control region is expected to have a properly functioning microcirculatory system with complex interactions, the tumor region may be ineffective. On the tumor side in figure 6.b. it can be seen that the side of the face closer to the nose and central in the tumor has more significant phase coherence locations than the side farther from this central area. The central part of the tumor mostly consists of necrotic tissue. Conversely, active neoangiogenesis is taking place in the tumor rim. Differences in blood flow regulation between these two regions are expected, especially because of the loose and irregular endothelium of tumor capillaries and therefore we would expect to see heterogeneous WPC within the tumor region.

\section{Conclusion}

We have presented a method for looking at connectivity in the microvasculature using time-frequency analysis of IR imaging to monitor the heat signature of blood flow changes. By using IR imaging with a sufficient sampling rate and timefrequency analysis we are able to analyze differences between locations in frequency content and phase coherence. We are also able to measure physiological vital signs including respiration rate, heart rate, and BTT using a non-contact IR camera. This leads to the study of real-time physiological temperature changes across time, frequency, and spatial locations using IR imaging.

\section{Acknowledgements}

This research was supported, in part, by the Intramural Research Program of the National Institute of Biomedical Imaging and Bioengineering, National Institutes of Health.

\section{REFERENCES}

[1] Bernjak A, Clarkson P B, McClintock P V and Stefanovska A, "Low-frequency blood flow oscillations in congestive heart failure and after beta1-blockade treatment," Microvasc Res, vol. 76, pp. 224-32, 2008.

[2] Bandrivskyy A, Bernjak A, McClintock P and Stefanovska A, "Wavelet Phase Coherence Analysis: Application to Skin Temperature and Blood Flow," Cardiovascular Engineering: An International Journal, vol. 4, pp. 89-93, 2004.

[3] Garbey M, Sun N, Merla A and Pavlidis I, "Contact-free measurement of cardiac pulse based on the analysis of thermal imagery," IEEE Trans Biomed Eng, vol. 54, pp. 1418-26, 2007.

[4] Gorbach A M, Wang H and Elster E, "Thermal oscillations in rat kidneys: an infrared imaging study," Philos Transact A Math Phys Eng Sci, vol. 366, pp. 3633-47, 2008.

[5] Gorbach A M, Heiss J, Kufta C, Sato S, Fedio P, Kammerer W A, Solomon J and Oldfield E H, "Intraoperative infrared functional imaging of human brain," Ann Neurol, vol. 54, pp. 297-309, 2003.

[6] Fukumura D, Duda D G, Munn L L and Jain R K, "Tumor microvasculature and microenvironment: novel insights through intravital imaging in pre-clinical models," Microcirculation, vol. 17, pp. 206-25, 2010.

[7] Haddadin A S, Abreu M M, Silverman D G, Luther M and Hines R L, "Noninvasive Assessment of Intracranial Temperature Via the Medial Canthal-Brain Temperature Tunnel," Anesthesiology, vol. 103, 2005.

[8] Neuro-science T W D o I, "SPM2 Toolbox," http://www.fil.ion.ucl.ac.uk/spm, London.

[9] Tankanag A and Chemeris N, "Application of the adaptive wavelet transform for analysis of blood flow oscillations in the human skin," Physics in Medicine and Biology, vol. 53, pp. 5967-5976, 2008.

[10] Torrence C and Compo G P, "A practical guide to wavelet analysis," Bulletin of the American Meteorological Society, vol. 79, pp. 61-78, 1998. 